\title{
A SUFFICIENT CONDITION FOR SURFACES IN 3-MANIFOLDS TO HAVE UNIQUE PRIME DECOMPOSITIONS
}

\author{
MICHAEL MOTTO
}

(Communicated by Ronald Stern)

\begin{abstract}
In 1975, Suzuki proved that prime decompositions of closed, connected surfaces in $S^{3}$ are unique up to ambient isotopy if the surface bounds a 3-manifold whose factors under the prime decomposition all have incompressible boundary. This paper extends this result to surfaces in more general 3-manifolds, when there is a prime decomposition for which every factor of the surface is incompressible on one side.
\end{abstract}

In 1975, Suzuki proved in [7] that prime decompositions of closed surfaces in $S^{3}$ are unique up to stable equivalence, and he also proved in [6] that this result could be strengthened to uniqueness up to ambient isotopy if the surface bounds a 3-manifold whose factors under the prime decomposition all have incompressible boundary. In [3] the former result was generalized to a large class of surfaces embedded in 3-manifolds. As a consequence of this and recent work of Scharlemann and Thompson [4], it is possible to generalize the latter result as well.

We start with a review of notation and definitions which will be needed. The term surface will refer here to a pair $(M, F)$, where $F$ is a compact 2manifold which is two-sided and properly embedded in a compact, connected 3-manifold $M$. A few special surfaces are a 2 -sphere embedded in the 3-sphere $\left(S^{3}, S^{2}\right)$, a disk properly embedded in a 3-ball $\left(B^{3}, D^{2}\right)$, and an unknotted torus embedded in the 3-sphere $\left(S^{3}, T^{2}\right)$. A connected sum of two surfaces, denoted $\left(M_{1}, F_{1}\right) \#\left(M_{2}, F_{2}\right)$, is formed by choosing 3-balls $B_{1}$ and $B_{2}$ in the interiors of $M_{1}$ and $M_{2}$, respectively, such that $\left(B_{i}, B_{i} \cap F_{i}\right) \cong\left(B^{3}, D^{2}\right)$ for $i=1,2$, removing the interiors of the $B_{i}$, and gluing together the remaining manifolds along a homeomorphism

$$
h:\left(\partial B_{1}, F_{1} \cap \partial B_{1}\right) \rightarrow\left(\partial B_{2}, F_{2} \cap \partial B_{2}\right) .
$$

This operation is in general not well defined, even up to homeomorphism, since it depends on both the choice of the component of $F_{i}$ which $B_{i}$ intersects and the choice of $h$ from among four possible isotopy classes. For example, if $\left(S^{3}, F\right)$ is a knotted torus, then depending on the choice of $h$, one could

Received by the editors July 23, 1992.

1991 Mathematics Subject Classification. Primary 57M99.

Key words and phrases. Heegaard splitting, prime decomposition, surface.

Supported in part by a grant from Ball State University. 
make $\left(S^{3}, F\right) \#\left(S^{3}, F\right)$ either a surface which bounds a genus 2 handlebody or a surface which bounds the union of a knotted solid torus and a cube-withknotted-hole. A decomposition, or factorization, of a surface $(M, F)$ is a representation of this surface as a connected sum

$$
(M, F)=\left(M_{1}, F_{1}\right) \#\left(M_{2}, F_{2}\right) \# \ldots \#\left(M_{n}, F_{n}\right),
$$

and the individual $\left(M_{i}, F_{i}\right)$ are called factors of the decomposition. A decomposition is nontrivial if no factor is $\left(S^{3}, S^{2}\right)$; a surface $(M, F)$ is prime if it is not $\left(S^{3}, S^{2}\right)$ and its only nontrivial decomposition is itself; and a decomposition is a prime decomposition if all of its factors are prime. Standard arguments show that every surface other than $\left(S^{3}, S^{2}\right)$ has a prime decomposition.

A stabilization of a surface $(M, F)$ is a connected sum

$$
(M, F) \#\left(S^{3}, T^{2}\right) \# \ldots \#\left(S^{3}, T^{2}\right),
$$

which may be viewed as a 2-manifold properly embedded in $M$. Two surfaces $\left(M_{1}, F_{1}\right)$ and $\left(M_{2}, F_{2}\right)$ are stably equivalent if there exists an (orientationpreserving) homeomorphism $h: M_{1} \rightarrow M_{2}$ and a surface $\left(M_{2}, F\right)$ which is a stabilization of both $\left(M_{2}, F_{2}\right)$ and $\left(M_{2}, h\left(F_{1}\right)\right)$.

A surface $(M, F)$ is said to possess unique prime decompositions up to stable equivalence if, given two prime decompositions

$$
\begin{aligned}
(M, F) & =\left(M_{1}, F_{1}\right) \# \ldots \#\left(M_{m}, F_{m}\right) \#\left(S^{3}, T^{2}\right) \# \ldots \#\left(S^{3}, T^{2}\right) \\
& =\left(M_{1}^{\prime}, F_{1}^{\prime}\right) \# \ldots \#\left(M_{n}^{\prime}, F_{n}^{\prime}\right) \#\left(S^{3}, T^{2}\right) \# \cdots \#\left(S^{3}, T^{2}\right),
\end{aligned}
$$

where no $\left(M_{i}, F_{i}\right)$ or $\left(M_{i}^{\prime}, F_{i}^{\prime}\right)$ is an $\left(S^{3}, T^{2}\right)$, it follows that $m=n$ and, after reordering if necessary, for $i=1, \ldots, n$ one has that $\left(M_{i}, F_{i}\right)$ and $\left(M_{i}^{\prime}, F_{i}^{\prime}\right)$ are stably equivalent. Note that the number of $\left(S^{3}, T^{2}\right)$ factors in each decomposition is not necessarily the same (since stable equivalence does not imply equal genus).

The main result of [3] is that surfaces $(M, F)$ have unique prime decompositions up to stable equivalence, provided that there is no nonseparating 2-sphere in $M$ which is eiiher disjoint from $F$ or which meets $F$ in a single simple closed curve, and that this result fails to hold in general if $M-F$ contains nonseparating 2-spheres. The case where $M$ contains nonseparating 2-spheres which meet $F$ in a single simple closed curve remains open. The result of Suzuki in [7] is the special case where $M=S^{3}$ and $F$ is connected. In order to generalize Suzuki's other result [6] which gives full uniqueness of the prime decompositions, we must first describe the notions of Heegaard splittings of manifolds with boundary and maximal triads of surfaces.

A 3-manifold triad is a triple $\left(M ; B_{1}, B_{2}\right)$ where $M$ is a compact 3manifold; $B_{1}$ and $B_{2}$ are disjoint (possibly empty) compact 2-manifolds contained in $\partial M$; and $\partial M-\left(B_{1} \cup B_{2}\right)$, if nonempty, is homeomorphic to $\partial B_{1} \times I$ with $\partial B_{1}=\partial B_{1} \times 0$ and $\partial B_{2}=\partial B_{1} \times 1$. A compression body is a triad $\left(W ; \partial_{-} W, \partial_{+} W\right)$ with $\partial_{+} W \neq \varnothing$, which has a decomposition into a collar of $\partial_{+} W$ together with 2-handles and 3-handles attached to this collar, and which furthermore has no 2-sphere components in $\partial_{-} W$. From a dual point of view, a compression body has a decomposition into a collar of $\partial_{-} W$, together with 0-handles and 1-handles attached to this collar. A compression body is a generalization of the notion of a handlebody and is in fact a handlebody if $\partial_{-} W=\varnothing$. 
A Heegaard splitting of a triad $\left(M ; B_{1}, B_{2}\right)$ is a surface $(M, F)$ such that $F$ is disjoint from $B_{1}$ and $B_{2}$, and $F$ separates $M$ into two compression bodies $W_{1}$ and $W_{2}$ where $F=\partial_{+} W_{1}=\partial_{+} W_{2}, B_{1}=\partial_{-} W_{1}$, and $B_{2}=\partial_{-} W_{2}$. This is equivalent to the classical definition of a Heegaard splitting in the case where $M$ is closed. The Reidemeister-Singer Theorem, which says that any two Heegaard splittings of a closed 3-manifold are stably equivalent, generalizes to the same result for 3-manifold triads [2, Chapter 4]. We say that a triad has unique Heegaard splittings if it possesses some Heegaard splitting such that every other Heegaard splitting is either ambient isotopic to, or a stabilization of, this one.

Given a surface $(M, F)$ such that $M-F$ is irreducible, one forms the maximal triad $T$ for $(M, F)$ as follows:

(1) start with a bicollar of $F$ in $M$,

(2) add 2-handles along a "maximal" set of compression disks of $F$,

(3) add 3-handles along sphere components of $\partial T-\partial M$, and

(4) if any component of $\partial T-\partial M$ is parallel to $\partial M$ in $M-F$, then thicken it to the boundary.

The process described above results in a triad $\left(T ; B_{1}, B_{2}\right)$ for which $(T, F)$ is a Heegaard splitting, $\partial T-\left(B_{1} \cup B_{2}\right)$ is contained in $\partial M$, and $B_{1}$ and $B_{2}$ are incompressible in $M$ - Int $T$. It turns out [3] that maximal triads are unique up to ambient isotopy with $F$ fixed, and that two surfaces $\left(M_{1}, F_{1}\right)$ and $\left(M_{2}, F_{2}\right)$ with $M_{i}-F_{i}$ irreducible are stably equivalent if and only if there is an (orientation-preserving) homeomorphism $h: M_{1} \rightarrow M_{2}$ which maps the maximal triad of one surface to the maximal triad of the other.

In seeking to strengthen uniqueness up to stable equivalence to uniqueness up to homeomorphism for prime decompositions in special cases, we will consider corresponding (stably equivalent) factors from two prime factorizations, and also their homeomorphic maximal triads. One case that needs special consideration occurs when corresponding factors $\left(M_{i}, F_{i}\right)$ and $\left(M_{i}^{\prime}, F_{i}^{\prime}\right)$ do not have $M_{i}-F_{i}$ or $M_{i}^{\prime}-F_{i}^{\prime}$ irreducible. However, it was shown in [3] that in this case either the 3-manifold factors are prime and $F_{i}$ and $F_{i}^{\prime}$ bound 3balls, or $M_{i}=M_{i}^{\prime}=S^{3}$ and $F_{i}$ and $F_{i}^{\prime}$ are each a disjoint union of two 2-spheres. Either way, there exists an (orientation-preserving) homeomorphism $h:\left(M_{i}, F_{i}\right) \rightarrow\left(M_{i}^{\prime}, F_{i}^{\prime}\right)$.

Theorem. Let $(M, F)$ be a surface such that no nonseparating 2-sphere of $M$ is disjoint from $F$ or meets it in a single simple closed curve. Suppose that $(M, F)$ has a prime decomposition such that every factor $\left(M_{i}, F_{i}\right)$ satisfies one of the following:

(1) $\left(M_{i}, F_{i}\right) \cong\left(S^{3}, T^{2}\right)$,

(2) $M_{i}-F_{i}$ is reducible, or

(3) the maximal triad of $\left(M_{i}, F_{i}\right)$ has unique Heegaard splittings.

Then this is the unique prime decomposition of $(M, F)$ up to homeomorphism. Proof. Consider a second prime decomposition of $(M, F)$. As was argued above, for every factor $\left(M_{i}, F_{i}\right)$ with $M_{i}-F_{i}$ reducible, there exists an (orientation-preserving) homeomorphism $h:\left(M_{i}, F_{i}\right) \rightarrow\left(M_{i}^{\prime}, F_{i}^{\prime}\right)$ to the corresponding factor of the second decomposition.

Next consider factors $\left(M_{i}, F_{i}\right)$ with $M_{i}-F_{i}$ irreducible and which are 
not homeomorphic to $\left(S^{3}, T^{2}\right)$. Since the corresponding factor $\left(M_{i}^{\prime}, F_{i}^{\prime}\right)$ is stably equivalent, there exists an (orientation-preserving) homeomorphism $h: M_{i}^{\prime} \rightarrow M_{i}$ which maps the maximal triad of one factor to that of the other. Since $F_{i}$ and $h\left(F_{i}^{\prime}\right)$ are Heegaard splittings of the maximal triad, which are not stabilizations since the factors are prime, and since the maximal triad has unique Heegaard splittings by hypothesis, it follows that $F_{i}$ and $h\left(F_{i}^{\prime}\right)$ are ambient isotopic in $M_{i}$. One may therefore assume without loss of generality that $h\left(F_{i}^{\prime}\right)=F_{i}$, and thus $h:\left(M_{i}, F_{i}\right) \rightarrow\left(M_{i}^{\prime}, F_{i}^{\prime}\right)$.

Finally, given the equivalence for these two types of factors, an obvious argument involving the Euler characteristic of $F$ shows that the two prime decompositions have an equal number of $\left(S^{3}, T^{2}\right)$ factors.

The following is a summary of the 3-manifolds which are currently known to have unique Heegaard splittings; in most cases, the relevant triad for a 3manifold $M$ is $(M ; \varnothing, \partial M)$ :

(1) $S^{3}[8]$

(2) $S^{2} \times S^{1}[8]$,

(3) lens spaces [1],

(4) $\left(W ; \partial_{-} W, \partial_{+} W\right)$ or $(W ; \varnothing, \partial W)$ where $W$ is an orientable compression body [4],

(5) $\left(W ; \partial_{-} W, \partial_{+} W\right)$ or $(W ; \varnothing, \partial W)$ where $W$ is a compression body and $\partial_{-} W$ is a 2 -manifold with boundary,

(6) handlebodies (compression bodies with $\left.\partial_{-} W=\varnothing\right)[1,8]$,

(7) $F^{2} \times I$ where $F^{2}$ is an orientable 2-manifold [4] or a 2-manifold which has boundary,

(8) $F^{2} \times S^{1}$ [5] where $F^{2}$ is an orientable 2-manifold.

Some additional comments should be made about a few of these cases.

The first two cases, $S^{3}$ and $S^{2} \times S^{1}$, are not relevant to the theorem which was stated above, since the only prime Heegaard splitting of $S^{3}$ is $\left(S^{3}, T^{2}\right)$ and since a factor which is a Heegaard splitting of $S^{2} \times S^{1}$ would allow a nonseparating 2-sphere which intersects the surface in a single simple closed curve.

The seventh case, $F^{2} \times I$, is a special case of the fourth and fifth cases, since $F^{2} \times I$ is a compression body. As a matter of fact, a standard technique used in the attempt to prove that compression bodies have unique Heegaard splittings is to cut the compression body along the cores of the 2-handles in some optimal 2-handle and 3-handle decomposition of the compression body, thus obtaining a manifold homeomorphic to $\partial_{-} W \times I$. One may then show that if $\partial_{-} W \times I$ has unique Heegaard splittings, then the original compression body $W$ also has unique Heegaard splittings. See, for example, [4, 2.7 and 2.12].

The sixth case is, in turn, a special case of the seventh, since every handlebody is homeomorphic to $F^{2} \times I$ for some 2-manifold with boundary $F^{2}$, where the handlebody will be orientable or nonorientable depending on whether $F^{2}$ is orientable or nonorientable. However, the proofs in [1] and [8] do not take this point of view; they give a direct proof that orientable handlebodies have unique Heegaard splittings. Although these results were only stated for orientable handlebodies, their arguments work equally well for nonorientable handlebodies. 
Some argument must still be made concerning the validity of the fifth case above, that a compression body $W$ has unique Heegaard splittings if $\partial_{-} W$ has boundary. As already discussed, this may be reduced to the question of whether $F^{2} \times I$ has unique Heegaard splittings when $\partial F^{2} \neq \varnothing$, whether $F^{2}$ is orientable or nonorientable. As indicated above, there are two ways of regarding the compression body $W$ as a triad, either $\left(W ; \partial_{-} W, \partial_{+} W\right)$ or $(W ; \varnothing, \partial W)$, and thus there are two types of Heegaard splittings to consider for compression bodies. These correspond to two types of Heegaard splittings to consider for $F^{2} \times I$. A Heegaard splitting of $\left(F^{2} \times I ; F^{2} \times 0, F^{2} \times 1\right)$ will separate the triad into two compression bodies, and the boundary of the Heegaard splitting may be assumed to be $\partial F^{2} \times(1 / 2)$. A Heegaard splitting of $\left(F^{2} \times I ; \varnothing, \partial\left(F^{2} \times I\right)\right)$ will be a closed surface which separates the triad into a compression body and an ordinary handlebody.

In the latter case, we are simply dealing with a handlebody $H=F^{2} \times I$ and, seeking its ordinary Heegaard splitting, that of the triad $(H ; \varnothing, \partial H)$. As was already mentioned above, arguments given in [1] and [8] to show that this has unique Heegaard splittings are valid in both the orientable and nonorientable cases, although they were only explicitly stated for the orientable case.

We must still argue the case that $\left(F^{2} \times I ; F^{2} \times 0, F^{2} \times 1\right)$ has unique Heegaard splittings when $F^{2}$ has boundary, whether $F^{2}$ is orientable or nonorientable. In $[4, \S 4]$, when $F^{2}$ is closed and there exists an arc $\gamma$ properly embedded in $F^{2} \times I$ which is vertical with respect to the Heegaard splitting in question (i.e., $h \uparrow \gamma$ has no critical points where $h$ is a Morse function corresponding to the Heegaard splitting), one proceeds as follows. Assume without loss of generality that $\gamma=x \times I$ for some point $x$ in $F^{2}$. Select a set of simple closed curves in $F^{2}$ which intersect only at the point $x$ and cut $F^{2}$ into a single polygon. Consider the union of annuli which is the product of these simple closed curves with $I$ in $F^{2} \times I$ and cuts $F^{2} \times I$ into an object called $G$, which is the product of a polygon and $I$. The Heegaard splitting meets each vertical edge of $G$ in exactly one point. The details of the proof take place primarily within $G$ and are in no way dependent on the orientability of $F^{2}$. The requirement of orientability is needed for other sections, when dealing with the case where the arc $\gamma$ is not vertical.

In the case we are currently concerned with, where $F^{2}$ has boundary and may or may not be orientable, one may select a set of disjoint arcs in $F^{2}$ which cut it into a single polygon. The disks which are the product of these arcs with $I$ then cut $F^{2} \times I$ into the product of a polygon and $I$, in which the Heegaard splitting will meet each vertical edge in exactly one point. The remainder of the proof then proceeds exactly as in $[4, \S 4]$.

Applying the above results on unique Heegaard splittings for compression bodies, whether $\partial_{-} W$ is closed or not, yields the following generalization of Suzuki's result in [6].

Corollary. Let $(M, F)$ be a surface with $M$ orientable such that no nonseparating 2-sphere of $M$ is disjoint from $F$ or meets it in a single simple closed curve. Suppose that $(M, F)$ has a prime decomposition such that every factor $\left(M_{i}, F_{i}\right)$ satisfies one of the following:

(1) $\left(M_{i}, F_{i}\right) \cong\left(S^{3}, T^{2}\right)$,

(2) $M_{i}-F_{i}$ is reducible, or 
(3) $F_{i}$ has no compression disk in $M_{i}$ on one side.

Then this is the unique prime decomposition of $(M, F)$ up to homeomorphism. Proof. This follows from the preceding theorem and the realization that, for any factor $\left(M_{i}, F_{i}\right)$, if $F_{i}$ has no compression disk in $M_{i}$ on one side, then its maximal triad will be a compression body (a thickened surface with 2-handles and 3-handles added to just one side), which will have unique Heegaard splittings.

It remains an open question whether this result generalizes to the nonorientable case, since one needs to prove the following.

Conjecture. Nonorientable compression bodies with $\partial_{-} W$ closed have unique Heegaard splittings.

Note that this conjecture can be reduced to the question of whether unique Heegaard splittings are possessed by triads $\left(F^{2} \times I ; F^{2} \times 0, F^{2} \times 1\right)$, where $F^{2}$ is a closed, nonorientable surface.

\section{REFERENCES}

1. F. Bonahon and J.-P. Otal, Scindements de Heegaard des espaces lenticulaires, Ann. Sci. École Norm. Sup. (4) 16 (1983), 451-466.

2. M. Motto, Surfaces in three-manifolds and three-manifold triads, Ph.D. dissertation, Univ. TexasAustin, 1988.

3. _ Maximal triads and prime decompositions of surfaces embedded in 3-manifolds, Trans. Amer. Math. Soc. 331 (1992), 851-867.

4. M. Scharlemann and A. Thompson, Heegaard splittings of (surface) $\times I$ are standard, Math. Ann. 295 (1993), 549-564.

5. J. Schultens, The classification of Heegaard splittings for (compact orientable surface) $\times S^{1}$, Proc. London Math. Soc. (3) 67 (1993), 425-448.

6. S. Suzuki, On surfaces in 3-sphere: prime decompositions, Hokkaido Math. J. 4 (1975), 179-195.

7. __ On surfaces in 3-sphere: stable congruence, Math. Japon. 20 (1975), 65-83.

8. F. Waldhausen, Heegaard-Zerlegungen der 3-Sphäre, Topology 7 (1968), 195-203.

Department of Mathematical Sciences, Ball State University, Muncie, Indiana 47306

E-mail address: 00mhmot to@leo.bsuvc.bsu.edu 